\title{
Follow-up study of airway microbiota in children with persistent wheezing
}

Lei $\mathrm{Wu}^{\dagger}$, Chencong Shen ${ }^{\dagger}$, Yuanling Chen, Xin Yang, Xiaofei Luo, Chengcheng Hang, Lingling Yan and Xuefeng $\mathrm{Xu}^{*}$ (1)

\begin{abstract}
Background: Increasing evidence revealed that airway microbial dysbiosis was associated with increased risk of asthma, or persistent wheezing (PW). However, the role of lung microbiota in PW or wheezing recurrence remains poorly understood.
\end{abstract}

Methods: In this prospective observational study, we performed a longitudinal $16 \mathrm{~S}$ rRNA-based microbiome survey on bronchoalveolar lavage (BAL) samples collected from 35 infants with PW and 28 age-matched infants (control group). A 2-year follow-up study on these PW patients was conducted. The compositions of lower airway microbiota were analyzed at the phylum and genus levels.

Results: Our study showed a clear difference in lower airway microbiota between PW children and the control group. Children with PW had a higher abundance of Elizabethkingia and Rothia, and lower abundance of Fusobacterium compared with the control group. At the end of the 2-year follow-up, 20 children with PW (57.1\%) experienced at least one episode of wheezing, and 15 (42.9\%) did not suffer from wheezing episodes. Furthermore, PW children with recurrence also had increased abundances of Elizabethkingia and Rothia relative to those who had no recurrence. Additionally, wheezing history, different gender, and caesarean section demonstrated a greater impact in airway microbiota compositions.

Conclusion: This study suggests that the alterations of lower airway microbiota could be strongly associated with the development of wheezing, and early airway microbial changes could also be associated with wheezing recurrence later in life.

Keywords: Airway, Children, Microbiota, Persistent wheezing

\section{Introduction}

Pediatric asthma is a common chronic disease of childhood and a major public health problem. Furthermore, asthma frequently begins in early childhood, and in up to half of asthma people, symptoms commence during childhood. Especially for children younger than

\footnotetext{
*Correspondence: xuxuefeng@zju.edu.cn

${ }^{\dagger}$ Lei Wu and Chencong Shen contribute equally to the work Department of Rheumatology Immunology \& Allergy, and Pulmonary Medicine, The Children's Hospital, Zhejiang University School of Medicine, National Clinical Research Center for Child Health, Hangzhou 310003, People's Republic of China
}

24 months, they usually manifest as repeated wheezing. Some infants will develop persistent or recurrent wheezing, which is often severe [1]. There is evidence that infantile wheezing or persistent wheezing (PW) is strongly associated with the development of asthma later in life [2]. However, the underlying mechanisms between them are still poorly understood. A number of biologically plausible mechanisms have suggested the effect of environmental changes early in life on the subsequent development of asthma, including cytokine response, developmental origins of adult disease, and microbial exposure [3-6]. 
The advances of culture-independent molecular technique of Next-Generation Sequencing (NGS) have confirmed the large number of microbiological organisms in the lungs of healthy individuals and patients with asthma [7]. The interactions between host and microbiota not only influence immune system development, but also play a significant role in regulating various inflammatory and metabolic pathways in the development of asthma [8, 9]. Furthermore, the airway microbial dysbiosis is associated with increased risk of asthma and asthma features $[10,11]$.

Currently, most studies on the role of airway microbiota in pediatric asthma or wheezing mainly focused on upper airway [12-15]. The data from lower airway samples might better reflect the connection between airway microbiota and the development of wheezing. A certain airway microbiota profile could be related with PW or wheezing recurrence in PW children. Here, we performed a $16 \mathrm{~S}$ rRNA-based microbiome survey on bronchoalveolar lavage (BAL) samples collected from children with PW, aiming to explore their airway microbiota compositions. At the same time, we also conducted a followup study on these PW patients, observing whether the recurrence of wheezing is related to the specific airway microbiota spectrum.

\section{Methods}

\section{Study design and BAL collection}

In this prospective observational cohort study, hospitalized children with PW were recruited from Children's Hospital of Zhejiang University School of Medicine. This study was approved by the Ethic Review Board of Children's Hospital, Zhejiang University School of Medicine (2018-IRB-069). Informed consent was obtained for all the subjects who are under 18 from a parent and/ or legal guardian. Diagnosis of persistent wheezing was based on persistent episodes of infantile wheezing [1]. In brief, inclusion criteria were as follow: (1) younger than 24 months; (2) duration of wheezing episode more than one month despite treatment with recommended first-line therapies of bronchodilators, inhaled corticosteroids, or systemic corticosteroids. All the included children were followed up to assess the recurrence of wheeze within 24 months of recruitment. The development of wheezing was confirmed by pediatrician, mainly based on wheezing symptoms and signs. At the same time, 28 age-matched infants underwent bronchoscopy due to bronchial granulation caused by foreign body aspiration, and these infants did not use any antibiotics and labeled as control group. Detailed clinical characteristics between children with PW and control subjects were listed in Table 1.
Table 1 Characteristics of children with persistent wheezing and control children

\begin{tabular}{|c|c|c|c|}
\hline & $\begin{array}{l}\text { Persistent } \\
\text { wheezing } \\
(n=35)\end{array}$ & Control $(n=28)$ & $P$ value \\
\hline Age (month) & $13.46 \pm 3.43$ & $15.64 \pm 5.31$ & 0.066 \\
\hline Gender (F/M) & $15 / 20$ & $12 / 16$ & 1.0 \\
\hline Cesarean section & 12 & 18 & $0.024^{*}$ \\
\hline WBC counts $\left(\times 10^{9} / \mathrm{L}\right)$ & $10.68 \pm 3.18$ & $10.53 \pm 2.92$ & 0.85 \\
\hline Neutrophils (\%) & $38.46 \pm 16.01$ & $33.11 \pm 8.57$ & 0.096 \\
\hline Eosinophils (\%) & $1.53 \pm 1.77$ & $2.12 \pm 1.71$ & 0.187 \\
\hline $\mathrm{Hgb}(\mathrm{g} / \mathrm{L})$ & $119.63 \pm 10.71$ & $118.82 \pm 10.14$ & 0.762 \\
\hline $\operatorname{PLT}\left(\times 10^{9} / \mathrm{L}\right)$ & $396.26 \pm 119.92$ & $375.11 \pm 136.21$ & 0.515 \\
\hline CRP (0-8 mg/L) & $3.85 \pm 8.00$ & $1.85 \pm 2.33$ & 0.206 \\
\hline $\mathrm{ALT}$ & $28.94 \pm 32.88$ & $18.21 \pm 6.92$ & 0.068 \\
\hline AST & $53.03 \pm 29.88$ & $47.46 \pm 23.09$ & 0.421 \\
\hline $\mathrm{LDH}$ & $404.97 \pm 175.41$ & $428.96 \pm 247.51$ & 0.654 \\
\hline
\end{tabular}

$W B C$ white blood cells, $\mathrm{Hg} b$ hemoglobin, $P L T$ blood platelet, $C R P C$ reactive protein, $A L T$ alanine aminotransferase, AST aspartate aminotransferase, $L D H$ lactate dehydrogenase

${ }^{*} P<0.05$, ${ }^{*} P<0.01$

Bronchoalveolar lavage fluid (BAL) collections were based on our previous study [16]. Bronchoscopy was transnasally performed using a flexible video-bronchoscope (XP260F, Olympus, Tokyo, Japan) following general anesthesia. To reduce irritation to the throat, local throat spray with lidocaine was performed. BAL was done by instillation of sterile saline $(1 \mathrm{ml} / \mathrm{kg}$, $\max 20 \mathrm{ml}$ ), and the collected BAL was centrifuged at $14000 \times g$ for $10 \mathrm{~min}$. The pellets were re-suspended in $500 \mu \mathrm{L}$ sterile PBS and stored at $-80{ }^{\circ} \mathrm{C}$ until DNA extraction.

\section{DNA extraction and 16S rRNA gene sequencing}

DNA extraction and 16S rRNA gene sequencing methods were based on our previous study [16]. Briefly, total genomic DNA was extracted using a commercial kit according to the manufacturer's instruction (DP382, Tiangen, Beijing, China). The extracted DNA were quantified with NanoDrop ND-1000 spectrophotometer (NanoDrop Technologies, Wilmington, DE, USA) and stored at $-80{ }^{\circ} \mathrm{C}$ until amplification. An aliquot of DNA from each BAL sample was used as the template for amplifications. To characterize the bacterial microbiota, the V3 to V4 regions of 16S rRNA gene were amplified (Forward primer 5'-CCTACGGGNGGC WGCAG-3' and Reverse primer 5'-GACTACHVGGG TATCTAATCC-3'), barcoded, and sequenced on the Illumina Miseq sequencing platform (Illumina, San Diego, USA). 


\section{Data processing and statistical analysis}

Quality filtering, denoising, and chimera removal were performed using the open-source software package, Quantitative Insights Into Microbial Ecology (QIIME, 1.9.13) platform [17]. Operational taxonomic units (OTUs) were defined using an identity cutoff value of $97 \%$ as this generally approximates the difference in $16 \mathrm{~S}$ sequences between bacterial species [18]. Taxa with the relative abundances $>0.1 \%$ was included in hierarchical clustering. The alpha-diversity estimates describe the number of species in a single sample, and it was calculated within QIIME using the Chaol and Shannon indexes. Taxonomy was assigned using the rdp-classifier-2.11 in QIIME (http://www.qiime.org) trimmed to sequences with complete taxonomic path. The rarefied sequence depth from 10 to the maximum value was set, and thirty points among them were determined. Rarefied samples were used to calculate alpha and beta diversity. The core microbiota was identified at the phylum and genus level.

All the statistical analysis and graphics were performed with $R$ statistical software packages ( $R$ version 3.4.3). Statistical analysis was performed using descriptive statistics. The continuous variables between groups were compared by Student's t-test or Mann-Whitney U test. Spearman rank correlations were used to demonstrate the association between various bacterial genera. For categorical variables, Pearson's chi-squared test was applied. A $P$ value of less than 0.05 was considered statistically significant in all the analysis.

\section{Results}

\section{Characteristics of included children}

During this study period, 35 children with PW were enrolled, and completed the follow-up (Table 1). Of them, 20 children $(57.1 \%)$ experienced at least one episode of wheezing (recurrence) at the end of the 2-year follow-up, and 15 (42.9\%) did not suffer from wheezing episodes (no recurrence). Compared with PW children without recurrence, PW children with recurrence had frequently a history of wheezing $(46.7 \%$ vs $95 \%, P=0.002$, Table 2). Although antibiotics administration had the impact on the airway microbiota, there were no significant differences observed in $\beta$-lactam and macrolides uses before hospitalization between the two groups.

\section{Airway microbiota composition of children with PW}

A total of 2,179,174 high-quality tags were generated, averaging 34,024 and 35,298 for the persistent wheezing and control groups, respectively. The average OTU numbers in the PW and control groups were 254 and 296 $(P=0.17)$, respectively. There were also no significant
Table 2 Characteristics of persistent wheezing children with and without recurrence

\begin{tabular}{|c|c|c|c|}
\hline & $\begin{array}{l}\text { Patients without } \\
\text { recurrence } \\
(n=15)\end{array}$ & $\begin{array}{l}\text { Patients with } \\
\text { recurrence } \\
(n=20)\end{array}$ & $P$ value \\
\hline Age (m) & $13.47 \pm 3.83$ & $13.45 \pm 3.20$ & 0.99 \\
\hline Gender (F/M) & $7 / 8$ & $13 / 7$ & 0.32 \\
\hline Cesarean section (n) & 6 & 6 & 0.72 \\
\hline Wheeze history (n) & 7 & 19 & $0.002^{* *}$ \\
\hline Follow-up time (m) & $\begin{array}{c}17.27 \pm 6.16 \\
(10-24)\end{array}$ & $\begin{array}{l}17.60 \pm 5.00 \\
(10-24)\end{array}$ & 0.86 \\
\hline $\begin{array}{l}\text { WBC counts } \\
\quad\left(\times 10^{9} / \mathrm{L}\right)\end{array}$ & $11.27 \pm 3.87$ & $10.23 \pm 2.56$ & 0.34 \\
\hline Neutrophils \% & $37.74 \pm 19.90$ & $39.00 \pm 12.89$ & 0.82 \\
\hline Eosinophils \% & $1.34 \pm 1.87$ & $1.68 \pm 1.73$ & 0.59 \\
\hline $\mathrm{Hgb}$ & $120.13 \pm 10.72$ & $119.25 \pm 10.96$ & 0.81 \\
\hline PLT & $386.47 \pm 90.91$ & $403.60 \pm 139.69$ & 0.68 \\
\hline $\mathrm{CRP}(0-8 \mathrm{mg} / \mathrm{L})$ & $3.92 \pm 10.60$ & $3.80 \pm 5.63$ & 0.96 \\
\hline $\mathrm{PCT}(<0.5 \mathrm{ng} / \mathrm{L})$ & $0.06 \pm 0.04$ & $0.08 \pm 0.07$ & 0.21 \\
\hline ALT & $35 \pm 45$ & $24 \pm 19$ & 0.35 \\
\hline AST & $61.13 \pm 36.87$ & $46.95 \pm 22.47$ & 0.17 \\
\hline $\mathrm{LDH}$ & $454.60 \pm 207.40$ & $367.75 \pm 141.29$ & 0.15 \\
\hline $\lg G$ & $6.35 \pm 1.45$ & $7.25 \pm 1.45$ & 0.08 \\
\hline $\lg A$ & $0.36 \pm 0.27$ & $0.53 \pm 0.23$ & 0.06 \\
\hline $\lg E$ & $106.32 \pm 192.22$ & $126.28 \pm 226.08$ & 0.79 \\
\hline
\end{tabular}

WBC white blood cells, $\mathrm{Hg} b$ hemoglobin, $P L T$ blood platelet, $C R P C$ reactive protein, $P C T$ procalcitonin, ALT alanine aminotransferase, AST aspartate aminotransferase, $L D H$ lactate dehydrogenase, $I g$ Immunoglobulin

${ }^{*} P<0.05$, ${ }^{*} P<0.01$

differences in the bacterial alpha diversity between the two groups using Chaol $(379 \pm 203$ vs $484 \pm 248$, $P=0.07)$ and Shannon indexes $(3.7 \pm 1.0$ vs $4.0 \pm 0.9$, $P=0.35)$.

A heatmap showed the airway microbiota composition at levels of phylum, class, order, family, and genus (Fig. 1). Overall, four dominant bacterial phyla were detected: Proteobacteria (35.5\% vs $39.6 \%, P=0.53$ ), Firmicutes (30.3\% vs $26.7 \%, P=0.52)$, Bacteroidetes $(20.6 \%$ vs $21.9 \%, P=0.75)$, and Actinobacteria $(4.2 \%$ vs $4.6 \%$, $P=0.80$ ) from children with $\mathrm{PW}$ and control group, respectively. Figure 2 showed a relative abundance of bacteria at the genus levels in each child. At the genus level, children with PW had a higher abundance of Elizabethkingia (2.6\% vs $0.5 \%, P=0.011)$ and Rothia $(1.5 \%$ vs $1.0 \%, P=0.27)$, and lower abundances of Moraxella $(2.9 \%$ vs $3.5 \%, \mathrm{p}=0.04)$ and Fusobacterium $(0.4 \%$ vs $2.3 \%$, $P=0.004)$ relative to control group.

\section{Effects of gender, delivery mode, and wheezing history on airway microbiota among children with PW}

No significant differences were observed in four dominant bacterial phyla proportions between boys and 


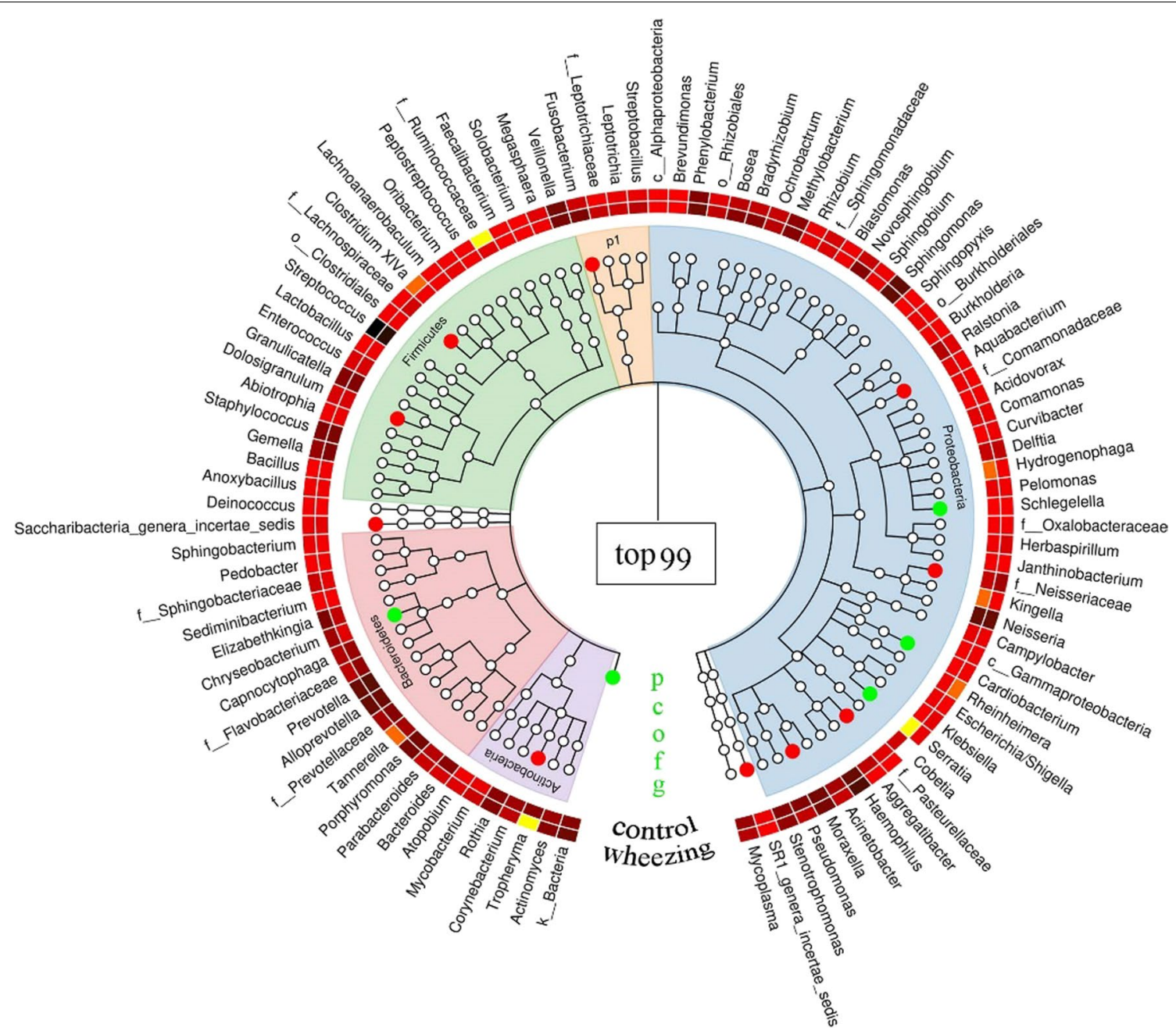

Fig. 1 A heatmap of the airway microbiota composition at levels of phylum (P), class (C), order (O), family (F), and genus (G). The innermost layer is the taxonomic tree labeled with background color, and the outermost layer is the annotated genus. The red globules indicated that there was significant difference between children with persistent wheezing and control group. The middle layer with red and yellow was a heatmap of average genus abundance, the darker the color, the higher the abundance

girls, vaginal delivery and cesarean section groups, and children with or without wheezing history. However, at the genus levels, boys with PW had lower abundances of Haemophilus, Prevotella, and Prophyromonas $(P<0.05$, respectively) compared with girls with $\mathrm{PW}$ (Fig. 3A). Relative to PW children with vaginal delivery, PW children with cesarean section had lower abundances of Streptococcus, Alloprevotella, Prevotella, and Rothia ( $\mathrm{P}<0.05$, respectively), and higher abundances of Sphingomonas, Elizabethkingia, and Phenylobacterium $(\mathrm{P}<0.05$, Fig. $3 \mathrm{~B})$. $\mathrm{PW}$ children with wheezing history had a higher abundance of Elizabethkingia, and a lower abundance of Haemophilus $(\mathrm{P}<0.05)$ compared with those without wheezing history. Despite no statistical difference, an increased abundance of Rothia was observed in PW children with wheezing history (Fig. 4A).

\section{Airway microbiota changes between PW children with and without recurrence}

To further understand the role of airway microbiota in the development of wheezing recurrence among children with PW, we performed a follow-up study. At the bacteria phylum level, no significant differences were observed with respect to the proportion of Proteobacteria, Firmicutes, Bacteroidetes, and Actinobacteria between children with and without recurrence. Notably at the genus level, PW children with recurrence had increased abundance of Elizabethkingia ( $4.3 \%$ vs $0.3 \%, P=0.002)$ and Rothia $(2.4 \%$ vs $0.3 \%, P=0.004)$, indicating that a different 


\section{a}
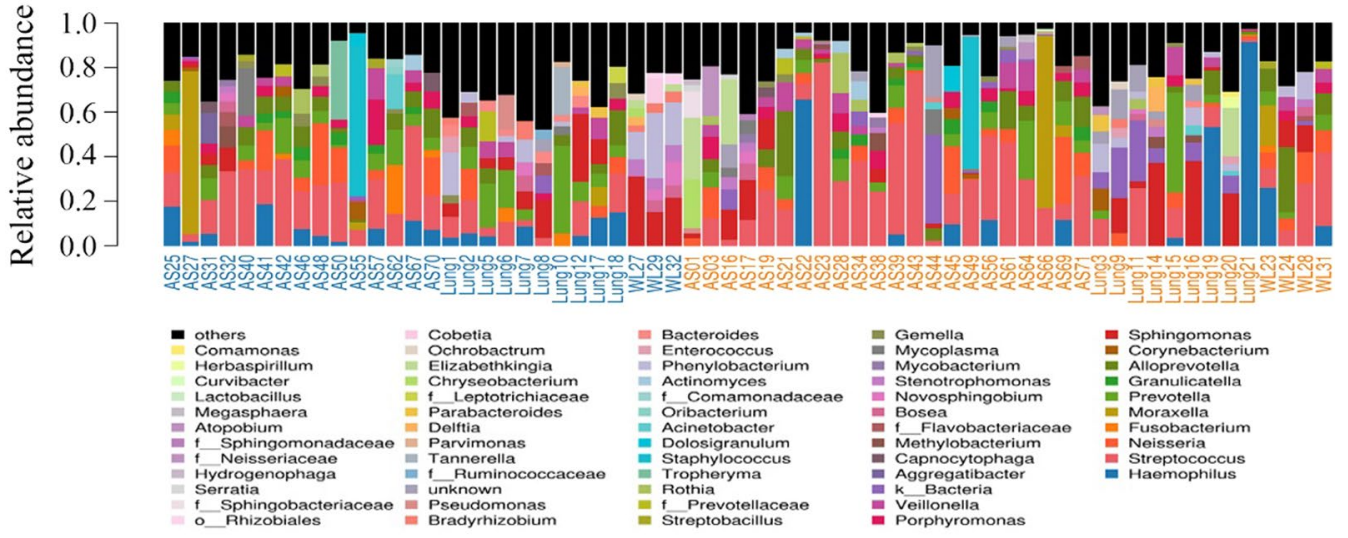

b
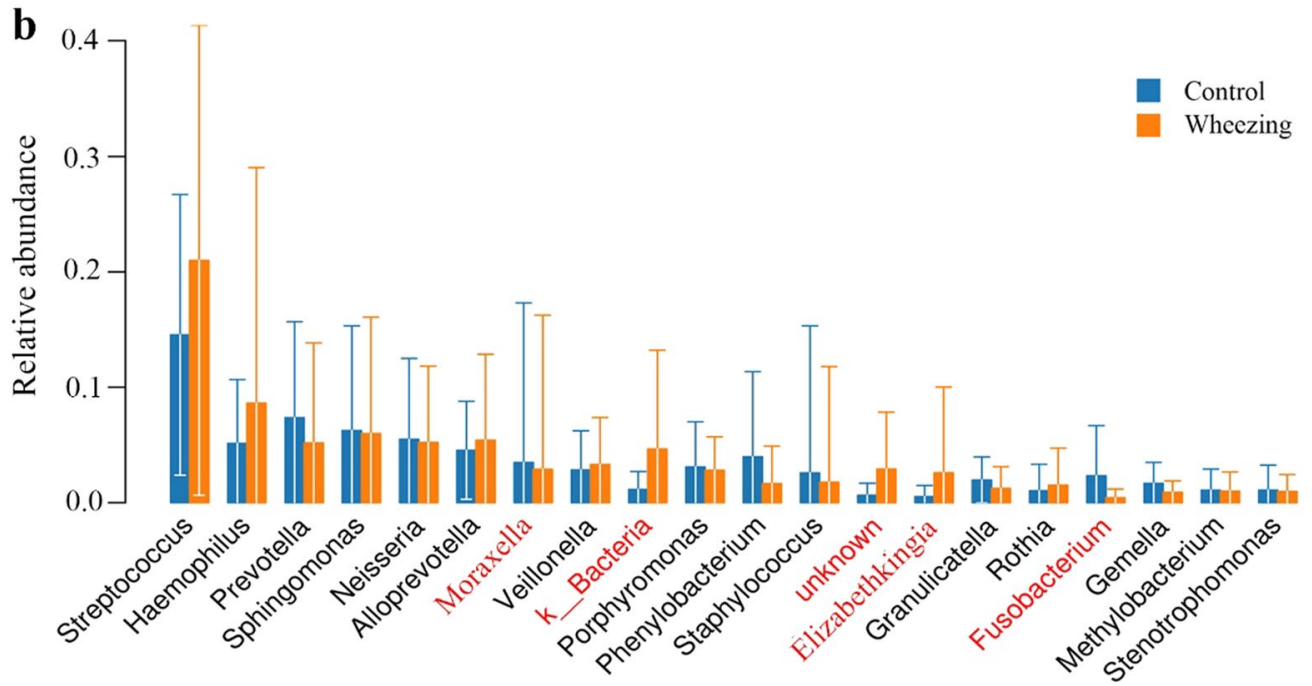

Fig. 2 Relative abundance plot of airway microbiota from children with persistent wheezing and control group. A showed a proportional abundance in each BAL sample at the genus level. B showed a relative abundance at the genus level between children with persistent wheezing and control group. The red-labeled bacterial genera represent significant differences $(P<0.05)$ between the two groups

airway microbiota profile might be associated with the recurrence of wheezing (Fig. 4B).

\section{Rank Correlation among the predominant genera in PW children}

Significant rank correlations between the predominant genera Elizabethkingia, Phenylobacterium, Porphyromonas, Rothia, Streptococcus, Veillonella, Haemophilus, Sphingomonas, Alloprevotella, Neisseria, and Prevotella were found among children with PW (Fig. 5A). The relative abundance of Elizabethkingia was positively correlated with abundances of Phenylobacterium $(\mathrm{r}=0.532, P=0.001)$ and Sphingomonas $(\mathrm{r}=0.735, \mathrm{P}<0.001)$, negatively correlated with abundances of Streptococcus $(\mathrm{r}=-0.398, P=0.018)$,
Veillonella $(\mathrm{r}=-0.409, \quad P=0.015)$, Alloprevotella $(\mathrm{r}=-0.448, P=0.007)$, and Prevotella $(\mathrm{r}=-0.429$, $P=0.01)$. The relative abundance of Rothia was positively correlated with abundances of Porphyromonas $(\mathrm{r}=0.519, \quad P=0.001), \quad$ Streptococcus $\quad(\mathrm{r}=0.536$, $P=0.001)$, and Prevotella $(\mathrm{r}=0.433, P=0.009)$, negatively correlated with abundances of Sphingomonas ( $\mathrm{r}=-0.411, P=0.014)$.

Additionally, in PW children with recurrence, the genus Elizabethkingia also showed similar correlation trend with abundances of Phenylobacterium, Sphingomonas, Streptococcus, Alloprevotella, and Prevotella. Although the relative abundance of Rothia was positively correlated with abundances of Porphyromonas $(\mathrm{r}=0.487, P=0.029)$, and staphylococcus $(\mathrm{r}=0.474$, 

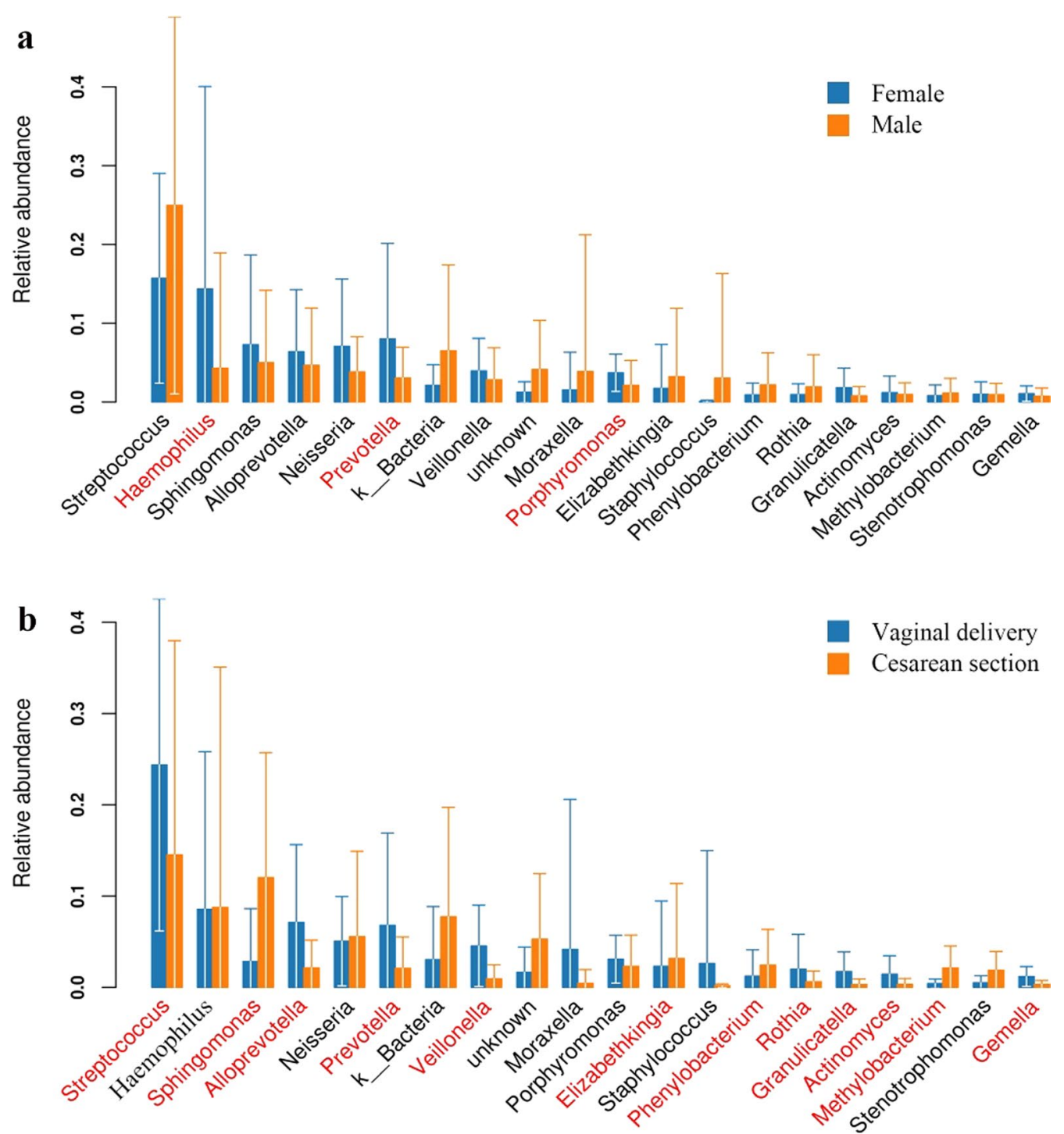

Fig. 3 Relative abundance plot of bacterial genus level from children with persistent wheezing. Bar graph A showed a difference between female and male groups, and $B$ showed a difference between vaginal delivery and cesarean section groups. The red-labeled bacterial genera represent significant differences $(P<0.05)$ between two groups

$P=0.035)$, Rothia indicated a weak correlation with Streptococcus $(\mathrm{r}=0.442, P=0.051$, Fig. 5B).

\section{Discussion}

This prospective study demonstrated a distinct characterization of airway microbiota in PW children younger than 24 months, and its associations with subsequent wheezing recurrence. We found that children with PW had higher abundances of Elizabethkingia and Rothia, and a lower abundance of Fusobacterium. Furthermore, during the follow-up period, $\mathrm{PW}$ children with recurrence also had increased abundances of Elizabethkingia and Rothia relative to those who had no recurrence. Although we did not find significant difference at the phylum level between children with PW and control group, the different dominant genera compositions were observed between the two groups. A previous study revealed that early Moraxella-dominant nasal microbiota profile in infants aged 2 months was associated with an increased rate of acute respiratory infections during the first 2 years of life [14]. The dominant Moraxella species of nasal microbiotas in older children with asthma were associated with increased exacerbation risk and 

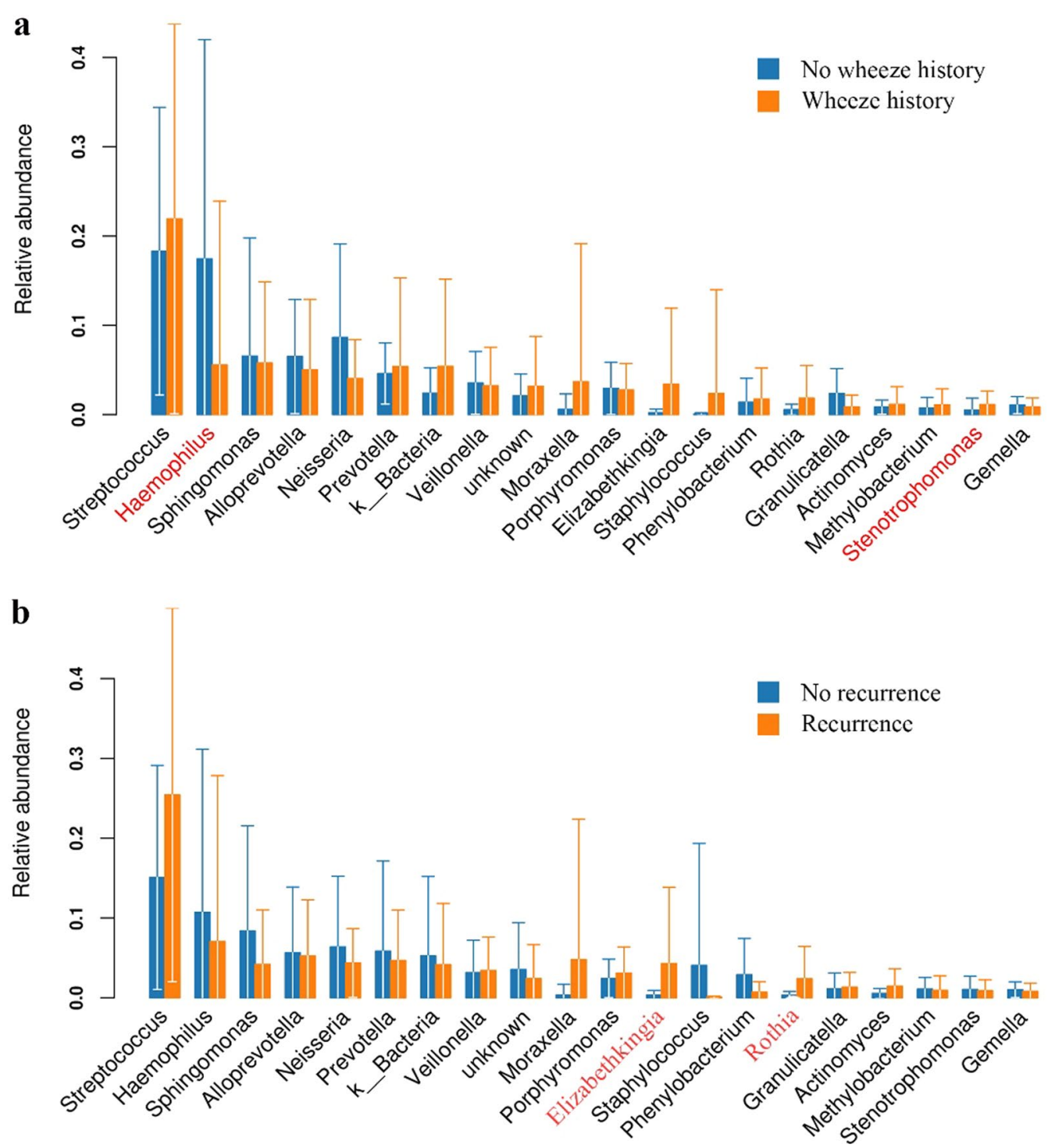

Fig. 4 Relative abundance plot of bacterial genus level from children with persistent wheezing. Bar graph A showed a difference between children with and without wheeze history, and B showed a difference between children with and without recurrence. The red-labeled bacterial genera represent significant differences $(P<0.05)$ between two groups

eosinophil activation [13]. This increased risk is likely to be correlated with significantly greater epithelial damage and inflammatory cytokine expression induced by Moraxella species [13]. The higher abundance of Haemophilus and Moraxella in airway microbiota might regulate airway inflammation during severe infantile bronchiolitis, potentially contributing to the development of subsequent wheezing recurrence in later childhood [3]. Moreover, dominant genera can vary with age. Older children had higher randomization abundance of Staphylococcus, while younger children had higher abundances of Moraxella and Streptococcus [4].
Different from the above studies, our study demonstrated that children with PW had higher abundances of Elizabethkingia and Rothia, and lower abundances of Moraxella and Fusobacterium in lower airway microbiota. This finding was similar to a previous study by Kazachkov et al. showing an enriched Rothia in lower airway microbiota from asthma children older than two years [19]. Members of the genus Elizabethkingia are gram-negative and nonmotile rods, and they can cause pneumonia, catheter-related bacteremia, neonatal meningitis, and even outbreaks $[20,21]$. So far, no report has been published regarding association 

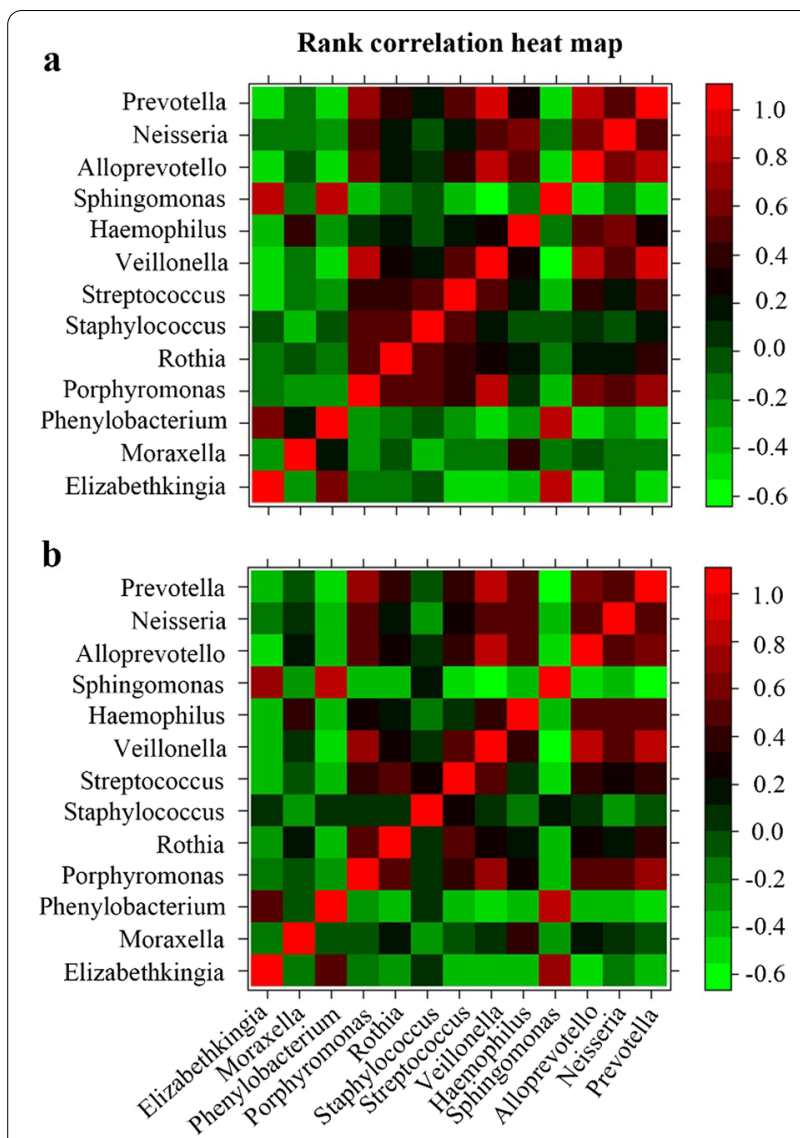

Fig. 5 Rank correlation heatmap between bacterial genus abundances in children with persistent wheezing. A showed a correlation matrix of bacterial genus abundances in children with persistent wheezing $(n=35)$, and B showed a correlation matrix in children with recurrence $(n=20)$. Red represents a positive correlation, and green indicates a negative correlation. Noted significant correlations of Elizabethkingia with Phenylobacterium, Streptococcus, Sphingomonas, Alloprevotella, and Prevotella, and Rothia with Porphyromonas, Staphylococcus, and Streptococcus

between Elizabethkingia and asthma or wheezing. It is likely that an increased abundance of Elizabethkingia might be involved in the pathogenesis of persistent wheezing. Nevertheless, whether overabundance of Elizabethkingia is the cause or consequence of persistent wheezing are not fully understood. Another increased trend of Rothia in lower airway microbiota was observed in PW children, although it was not statistically significant. Additionally, PW children with wheezing history also possessed higher abundances of Elizabethkingia and Rothia. These findings strongly suggested a close association between increased abundances of Elizabethkingia and Rothia and infantile wheezing. However, PW children showed a lower abundance of Moraxella, which could be associated with different age and sampling location. Although PW children with recurrence had a higher abundance of Moraxella compared with those without recurrence, there was no significant difference between the two groups. This difference still needs to be further investigated to confirm. On the other hand, we also found significant rank correlations between Elizabethkingia or Rothia and other predominant genera Phenylobacterium, Porphyromonas, Streptococcus, Veillonella, Haemophilus, Sphingomonas, Alloprevotella, Neisseria, and Prevotella. The rank correlations among the predominant genera indicated that specific microbiota profiles could be associated with the development or progression of infantile wheezing. However, the underlying mechanisms still need further investigation.

Notably in this study, different gender and delivery mode also illustrated a greater impact in airway microbiota compositions, revealing the complexity of the formation of airway flora. Although overall gut microbial community structure at age 2-4 years was not associated with preschool wheezing or future asthma development at age 6 [22], our study on lower airway microbiota demonstrated a correlation between them. Interestingly in the present 2-year follow-up, we found that PW children who had at least one episode of wheezing recurrence also had increased abundances of Elizabethkingia and Rothia relative to those who had no recurrence. It further indicated that there is a clear correlation between airway microbiota and wheezing recurrence. This early specific alterations in airway microbiota are likely to indicate an increased risk of wheezing recurrence. Nevertheless, whether airway microbial alteration is the cause or consequence of wheezing needs further investigation. On the other hand, lung microbiota might be affected by many external factors. Environmental factors such as bed dust could influence airway microbiota compositions among infants [23]. Maternal dietary intervention with longchain fatty acids and vitamin $\mathrm{D}$ supplementation can affect the infant airways but not the infant fecal microbiota [24]. Additionally, other potential confounders including living areas and living conditions can also have a certain impact on airway microbiota. This will further complicate the airway microbiota research.

Currently, 16S rRNA gene amplicon analysis remains the standard approach for the cultivation independent investigation of microbiota, and the accuracy of these analyses depends mainly on primer selections [25]. The nine hypervariable regions (V1-V9) of 16S rRNA gene are frequently used for determining of the bacterial taxonomy in the diverse microbial populations [4, 14, 23]. In general, high-throughput short-read sequencing of the 16S rRNA gene amplicon based on the Illumina MiSeq platform specifically targets the V3-V4 hypervariable 
region [26]. This region provides ample information for taxonomic classification of microbial communities from specimens associated with human microbiome studies [27]. However, due to the limitations of the technology itself, we did not definitely detect the species. Although bacterial culturing was routinely performed for BAL specimens, we failed to find the pathogenic bacteria or the same bacteria as those detected by $16 \mathrm{~S}$ rRNA sequencing method. This phenomenon may be associated with less microbial ratios or antibiotic use before hospitalization. Now, a new 16S full-length-based synthetic long-read sequencing technology can be a choice to read the whole variable regions of $16 \mathrm{~S}$ rRNA gene to identify the microbial communities in metagenome studies [26].

The present study has some limitations that should be considered. First, our study mainly focused on bacterial abundance via 16S rRNA analysis, not involved in the investigations of virus and fungi. A comprehensive analysis of bacterial, viral, and fungal microbiota would contribute us to better understanding host-microbiota interaction. Second, the influences of many other factors that might induce airway dysbiosis, are difficult to exclude, including corticosteroid and antibiotic uses. Although antibiotics have a major role in airway microbiota composition, antibiotic use will be inevitable for children with persistent wheezing in China. All the presently included children with persistent wheezing had varying degrees of antibiotic use. The rate of antibiotic treatments was similar between the persistent wheezing children with and without recurrence. Since bronchoscopy was performed via the nose, BAL samples would inevitably be contaminated. However, we followed standard bronchoscopy procedure for each patient to minimize the risk of contamination.

\section{Conclusion}

The overabundances of Elizabethkingia and Rothia in the BAL of children with PW suggest that the imbalance of airway microbiota could be associated with the development of wheezing. Moreover, this early airway microbial alteration could also be correlated with wheezing recurrence later in life. In addition, the potential role of early airway microbial alteration in pathogenesis of children wheezing will need further investigation.

\section{Abbreviations}

BAL: Bronchoalveolar lavage; OTU: Operational taxonomic units; PW: Persistent wheezing; QIIME: Quantitative insights into microbial ecology; rRNA: Ribosomal RNA.

Acknowledgements

Not applicable.

\section{Authors' contributions}

All authors contributed to the development of the selection criteria, the risk of bias assessment strategy and data extraction criteria. XXF is the guarantor. WL, SCC, and XXF drafted the manuscript. XXF, YX, and LXF developed the search strategy. HCC and YLL provided statistical expertise. CYL reviewed and revised manuscript. All authors read and approved the final manuscript.

\section{Funding}

This work was supported by fund from the National Natural Science Foundation of China (81871220). The funder had no role in study design, data collection and interpretation, or the decision to submit the work for publication.

Availability of data and materials

The datasets used and/or analyzed during the current study are available from the corresponding author on reasonable request.

\section{Declarations}

\section{Ethics approval and consent to participate}

This study was approved by the Ethic Review Board of Children's Hospital, Zhejiang University School of Medicine (2018-IRB-069). Informed consent was obtained for all the subjects who are under 18 from a parent and/or legal guardian.

\section{Consent for publication}

Not applicable.

\section{Competing interests}

The authors declare that they have no competing interests.

Received: 1 May 2021 Accepted: 19 July 2021

Published online: 27 July 2021

References

1. Ren CL, Esther CR, Debley JS, Sockrider M, Yilmaz O, Amin N, Bazzy-Asaad A, Davis SD, Durand M, Ewig JM, et al. Official American thoracic society clinical practice guidelines: diagnostic evaluation of infants with recurrent or persistent wheezing. Am J Respir Crit Care Med. 2016;194(3):356-73.

2. Just J, Bourgoin-Heck M, Amat F. Clinical phenotypes in asthma during childhood. Clin Exp Allergy. 2017:47(7):848-55.

3. Zhang X, Zhang X, Zhang N, Wang X, Sun L, Chen N, Zhao S, He Q. Airway microbiome, host immune response and recurrent wheezing in infants with severe respiratory syncytial virus bronchiolitis. Pediatr Allergy Immunol. 2020;31(3):281-9.

4. Zhou Y, Jackson D, Bacharier LB, Mauger D, Boushey H, Castro M, Durack J, Huang Y, LemanskeStorch RFGA, et al. The upper-airway microbiota and loss of asthma control among asthmatic children. Nat Commun. 2019;10(1):5714.

5. Sheng YJ, Xu SS, Li XJ, Liu JL, Wu XL, Xu XF. Low birth weight contributed to increased serum IL-6 levels in infantile respiratory syncytial virus infection. BMC Pediatr. 2017;17(1):205.

6. Xu XF, Hu QY, Liang LF, Wu L, Gu WZ, Tang LL, Fu LC, Du LZ. Epigenetics of hyper-responsiveness to allergen challenge following intrauterine growth retardation rat. Respir Res. 2014;15:137.

7. Faner R, Sibila O, Agusti A, Bernasconi E, Chalmers JD, Huffnagle GB, Manichanh C, Molyneaux PL, Paredes R, Perez Brocal V, et al. The microbiome in respiratory medicine: current challenges and future perspectives. Eur Respir J. 2017;49:4.

8. Man WH, de Steenhuijsen Piters WA, Bogaert D. The microbiota of the respiratory tract: gatekeeper to respiratory health. Nat Rev Microbiol. 2017;15(5):259-70.

9. Chung KF. Airway microbial dysbiosis in asthmatic patients: A target for prevention and treatment? J Allergy Clin Immunol. 2017;139(4):1071-81.

10. Goleva E, Jackson LP, Harris JK, Robertson CE, Sutherland ER, Hall CF, Good JT Jr, Gelfand EW, Martin RJ, Leung DY. The effects of airway microbiome on corticosteroid responsiveness in asthma. Am J Respir Crit Care Med. 2013;188(10):1193-201. 
11. Abdel-Aziz MI, Vijverberg SJH, Neerincx AH, Kraneveld AD. Maitland-van der Zee AH. The crosstalk between microbiome and asthma: exploring associations and challenges. Clin Exp Allergy. 2019;49(8):1067-86.

12. Teo SM, Tang HHF, Mok D, Judd LM, Watts SC, Pham K, Holt BJ, Kusel M, Serralha M, Troy N, et al. Airway microbiota dynamics uncover a critical window for interplay of pathogenic bacteria and allergy in childhood respiratory disease. Cell Host Microbe. 2018;24(3):341-352.e345.

13. McCauley K, Durack J, Valladares R, Fadrosh DW, Lin DL, Calatroni A, LeBeau PK, Tran HT, Fujimura KE, LaMere B, et al. Distinct nasal airway bacterial microbiotas differentially relate to exacerbation in pediatric patients with asthma. J Allergy Clin Immunol. 2019;144(5):1187-97.

14. Toivonen L, Hasegawa K, Waris M, Ajami NJ, Petrosino JF, Camargo CA Jr, Peltola V. Early nasal microbiota and acute respiratory infections during the first years of life. Thorax. 2019;74(6):592-9.

15. Pérez-Losada M, Authelet KJ, Hoptay CE, Kwak C, Crandall KA, Freishtat RJ. Pediatric asthma comprises different phenotypic clusters with unique nasal microbiotas. Microbiome. 2018;6:15.

16. Xu X, Wu L, Sheng Y, Liu J, Xu Z, Kong W, Tang L, Chen Z. Airway microbiota in children with bronchial mucus plugs caused by Mycoplasma pneumoniae pneumonia. Respir Med. 2020;170:105902.

17. Caporaso JG, Kuczynski J, Stombaugh J, Bittinger K, Bushman FD, Costello EK, Fierer N, Pena AG, Goodrich JK, Gordon Jl, et al. QIIME allows analysis of high-throughput community sequencing data. Nat Methods. 2010;7(5):335-6.

18. Janda JM, Abbott SL. 16 S rRNA gene sequencing for bacterial identification in the diagnostic laboratory: pluses, perils, and pitfalls. J Clin Microbiol. 2007;45(9):2761-4.

19. Kazachkov M, Kapoor BC, Malecha PW, Wu BG, Li Y, Levine J, Erkman J, Fitzgerald K, Moy L, Segal LN. Aerodigestive dysbiosis in children with chronic cough. Pediatr Pulmonol. 2018;53(9):1288-98.

20. Jean SS, Lee WS, Chen FL, Ou TY, Hsueh PR. Elizabethkingia meningoseptica: an important emerging pathogen causing healthcare-associated infections. J Hosp Infect. 2014;86(4):244-9.
21. Janda JM, Lopez DL. Mini review: new pathogen profiles: Elizabethkingia anophelis. Diagn Microbiol Infect Dis. 2017;88(2):201-5.

22. Bannier M, van Best N, Bervoets $L$, Savelkoul PHM, Hornef MW, van de Kant KDG, Jobsis Q, Dompeling E, Penders J. Gut microbiota in wheezing preschool children and the association with childhood asthma. Allergy. 2020;75(6):1473-6.

23. Gupta S, Hjelmso MH, Lehtimaki J, Li X, Mortensen MS, Russel J, Trivedi U, Rasmussen MA, Stokholm J, Bisgaard H, Sorensen SJ. Environmental shaping of the bacterial and fungal community in infant bed dust and correlations with the airway microbiota. Microbiome. 2020;8(1):115.

24. Hjelmso MH, Shah SA, Thorsen J, Rasmussen M, Vestergaard G, Mortensen MS, Brejnrod A, Brix S, Chawes B, Bonnelykke K, et al. Prenatal dietary supplements influence the infant airway microbiota in a randomized factorial clinical trial. Nat Commun. 2020;11(1):426.

25. Klindworth A, Pruesse E, Schweer T, Peplies J, Quast C, Horn M, Glockner FO. Evaluation of general $16 \mathrm{~S}$ ribosomal RNA gene PCR primers for classical and next-generation sequencing-based diversity studies. Nucleic Acids Res. 2013;41(1):e1.

26. Jeong J, Yun K, Mun S, Chung WH, Choi SY, Nam YD, Lim MY, Hong CP, Park C, Ahn YJ, Han K. The effect of taxonomic classification by full-length $16 \mathrm{~S}$ rRNA sequencing with a synthetic long-read technology. Sci Rep. 2021;11(1):1727.

27. Fadrosh DW, Ma B, Gajer P, Sengamalay N, Ott S, Brotman RM, Ravel J. An improved dual-indexing approach for multiplexed 16S rRNA gene sequencing on the Illumina MiSeq platform. Microbiome. 2014;2(1):6.

\section{Publisher's Note}

Springer Nature remains neutral with regard to jurisdictional claims in published maps and institutional affiliations.
Ready to submit your research? Choose BMC and benefit from:

- fast, convenient online submission

- thorough peer review by experienced researchers in your field

- rapid publication on acceptance

- support for research data, including large and complex data types

- gold Open Access which fosters wider collaboration and increased citations

- maximum visibility for your research: over $100 \mathrm{M}$ website views per year

At BMC, research is always in progress.

Learn more biomedcentral.com/submissions 